\title{
Efficacy of Difelikefalin in Subjects With Moderate-to-Severe Chronic Kidney Disease-Associated Pruritus: Pooled Subgroup Analysis of KALM-1 and KALM-2
}

Joel Topf'; Warren Wen²; Catherine Munera²; Frédérique Menzaghi²; Michael Schömig ${ }^{3}$ 'St. Clair Nephrology, Roseville, Ml; ${ }^{2}$ Cara Therapeutics, Stamford, CT; ${ }^{3}$ Dialysezentrum Heilbronn, Heilbronn, Germany

SYNOPSIS

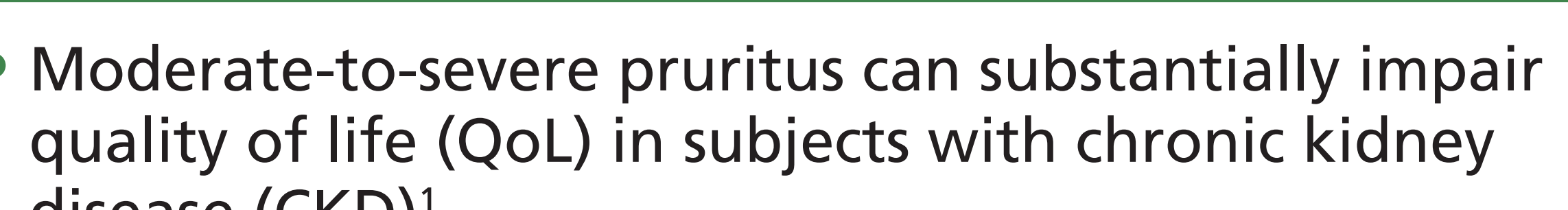

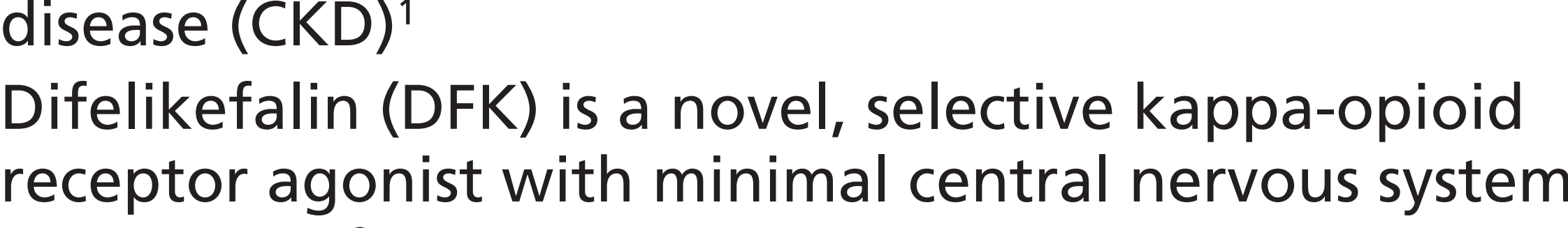
receptor agonist with minimal central nerrous syst
penentration - In August 2021, intravenous (IV) DFK received
approvol from the US Food and Drug Administration for the treatment of moderate-t--5evere pruritus
associated with chronic kidney disease in adults undergoing hemodialysis (HD) Together, the KALM-1 - and KALM-2 studies comprise
the largest global phase 3 program conducted in subjects

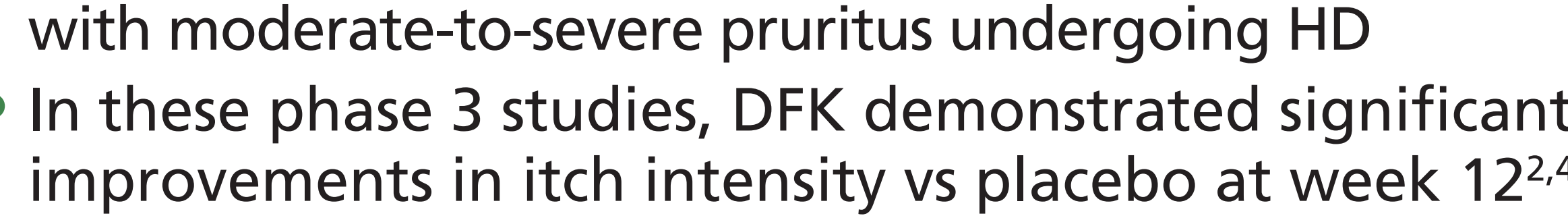
OBJECTIVE

To further the understanding of the efficacy profile
of $D F K$, we report efficacy and Qol outcomes in

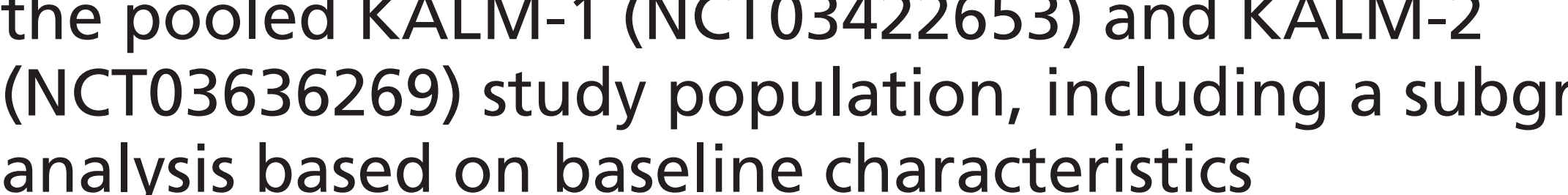
METHODS

KALM-1 and KALM-2 were randomized, phase 3 ,
multicenter, placebo-controlled studies (Figure 1) KALL-1 was conducted in the United States, and
KALM-2 was conducted in the United States, canada,
Europe, Asia, Australia, and New Zealand subjects with moderate to severe CKD-associaten pruritus (CKD-aP) undergoing HD were randomized
to VI DFK $0.5 \mathrm{mcg} / \mathrm{kg}$ or placebo 3 times/week for
12 weeks

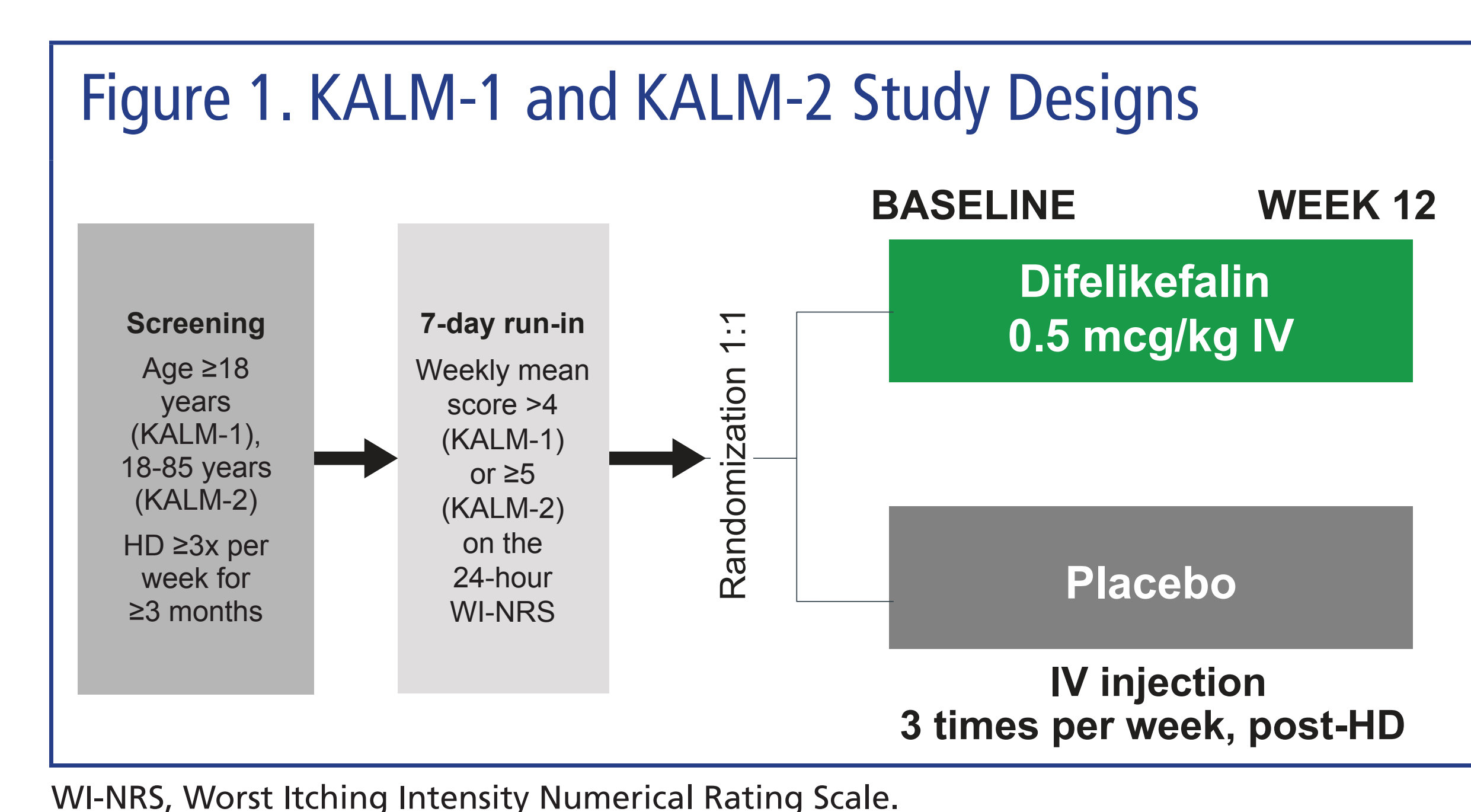

Outcomes

The primary endpoint in KALM-1 and KALM-2 was the
percentage of subjects achieving $23-$-point i improvement
at week 12 in weekly mean of dist at week 12 in weekly mean of daily WI-NRS Score
Achievement of $>3$-point improvement in weekly

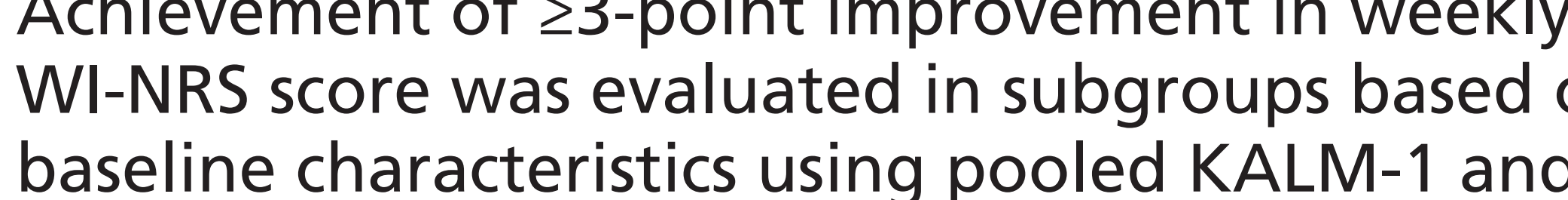
baseline charactse
KALM-2 data

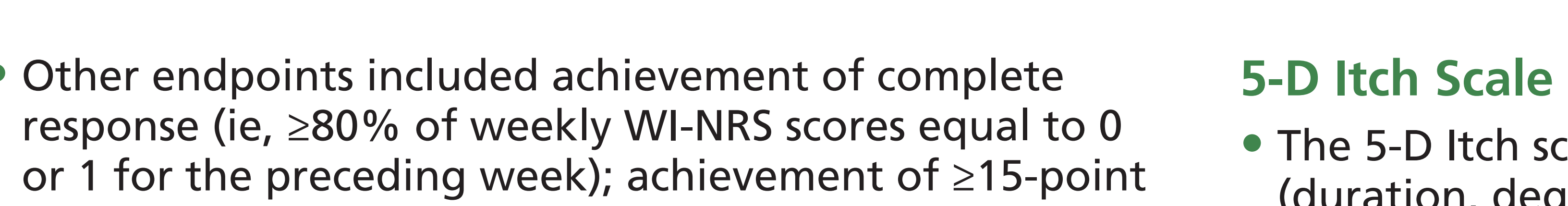

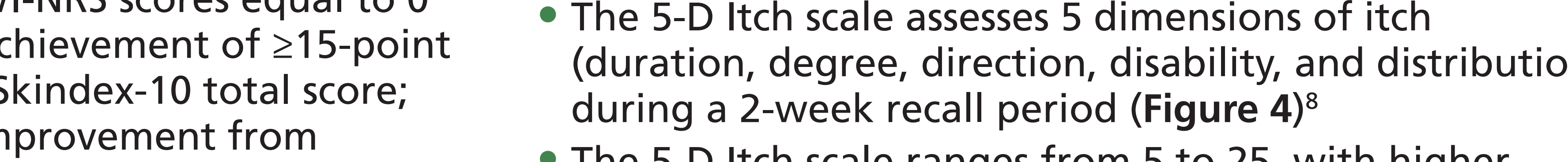
rovement from baseline in 5 -D Itch total score

Worst Itching Intensity Numerical Rating Scale -The WI-NRS is a validated 11-point scale ranging from 0

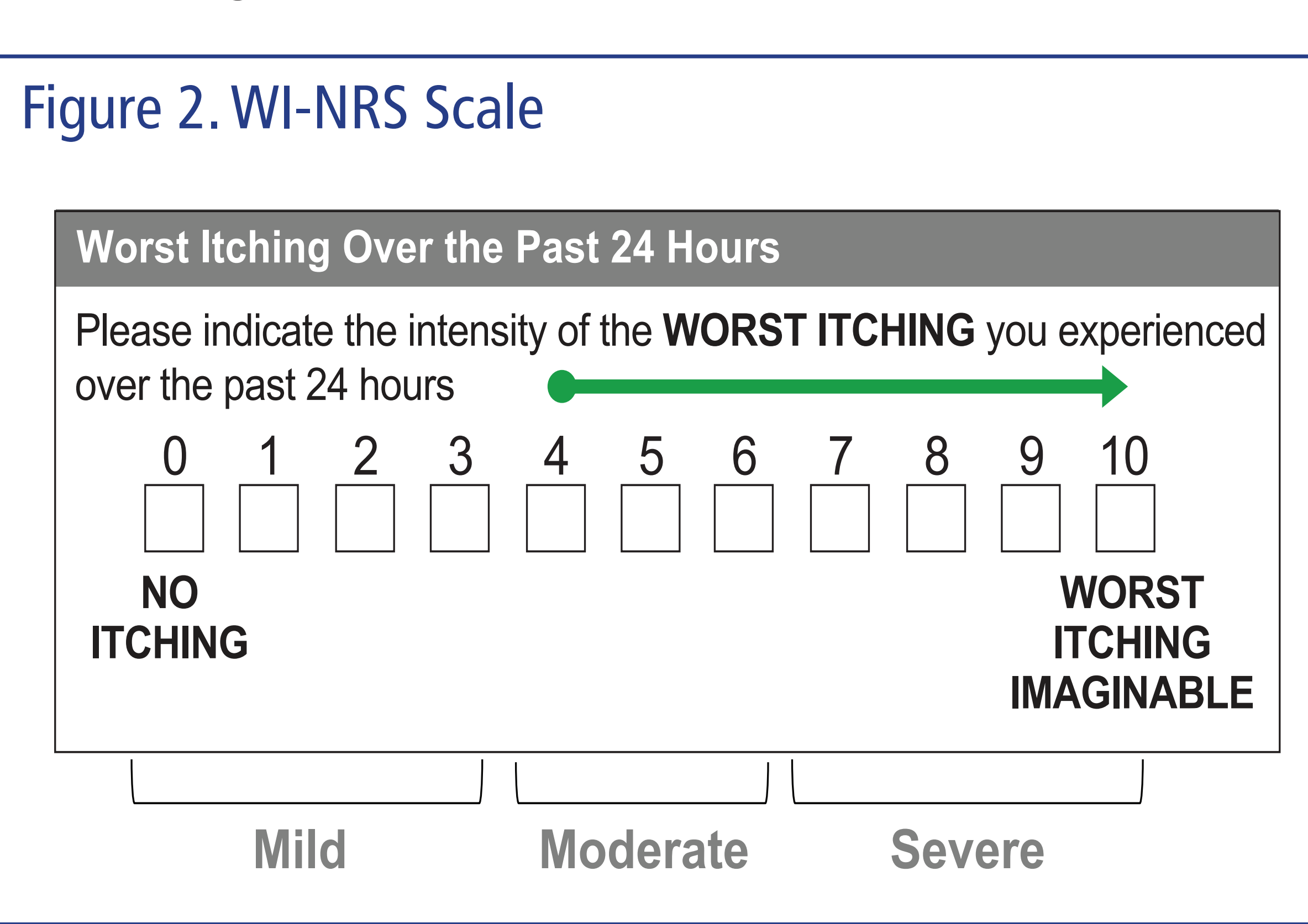
Reduction of $\geq 3$ points on the WI-NRS is associated with
cliniclly meaningfulchange in itth severity for patients
with moderate-to-severe CKD-AP? - The Skindex-10 scale was developed specifically for
assessing itch-related Qol across 3 domains in $\mathrm{HD}$ Higher total Skindex-10 scores indicate worse itcSkindex-10 Scale Figure 3. Skindex-10 Scale

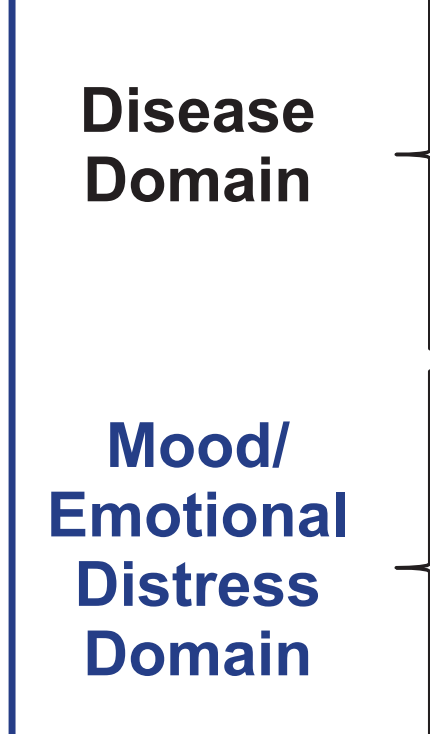
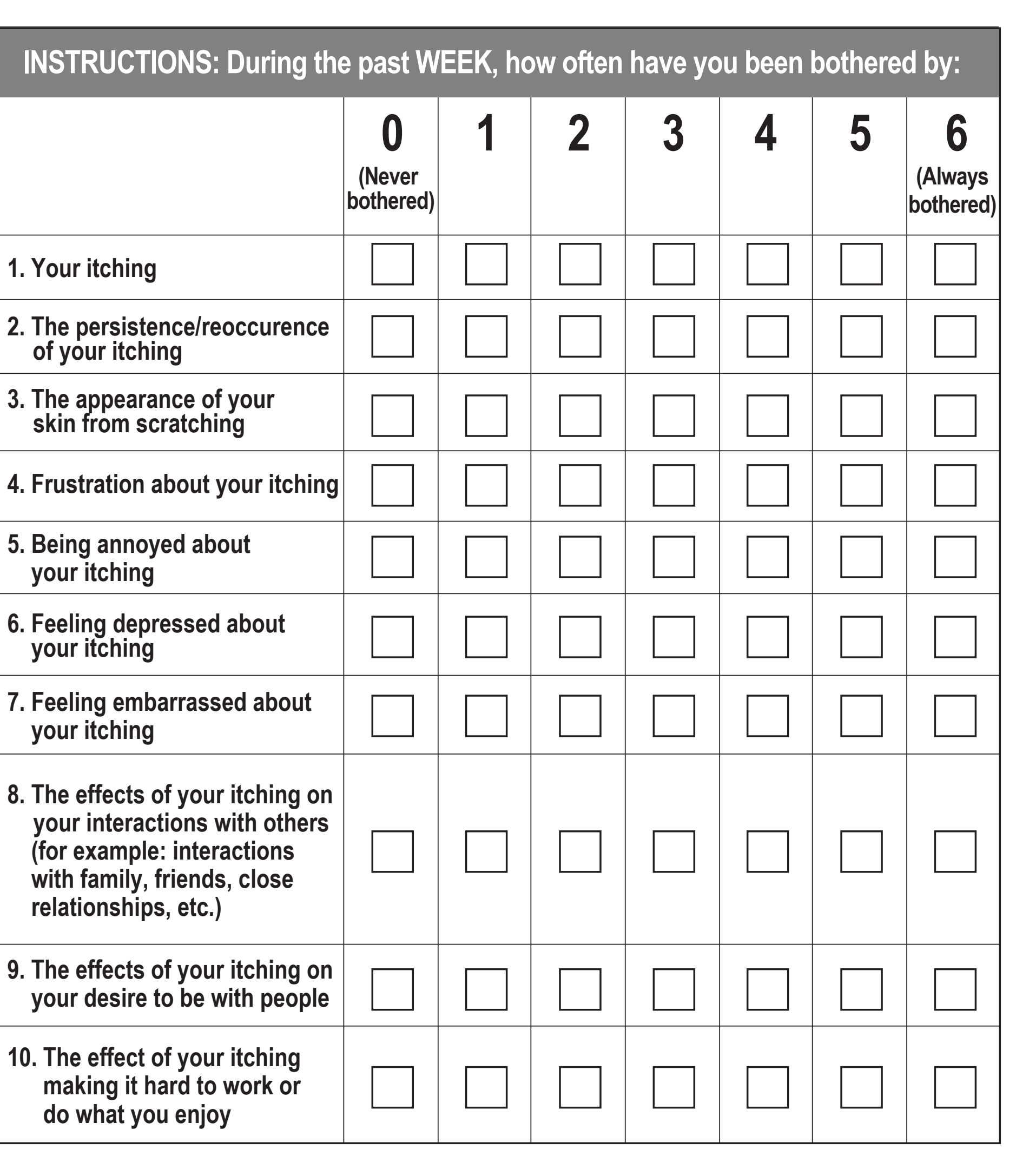
scores indicating worse itch-related Qo ${ }^{8}$

Figure 4.5-D Itch Scale

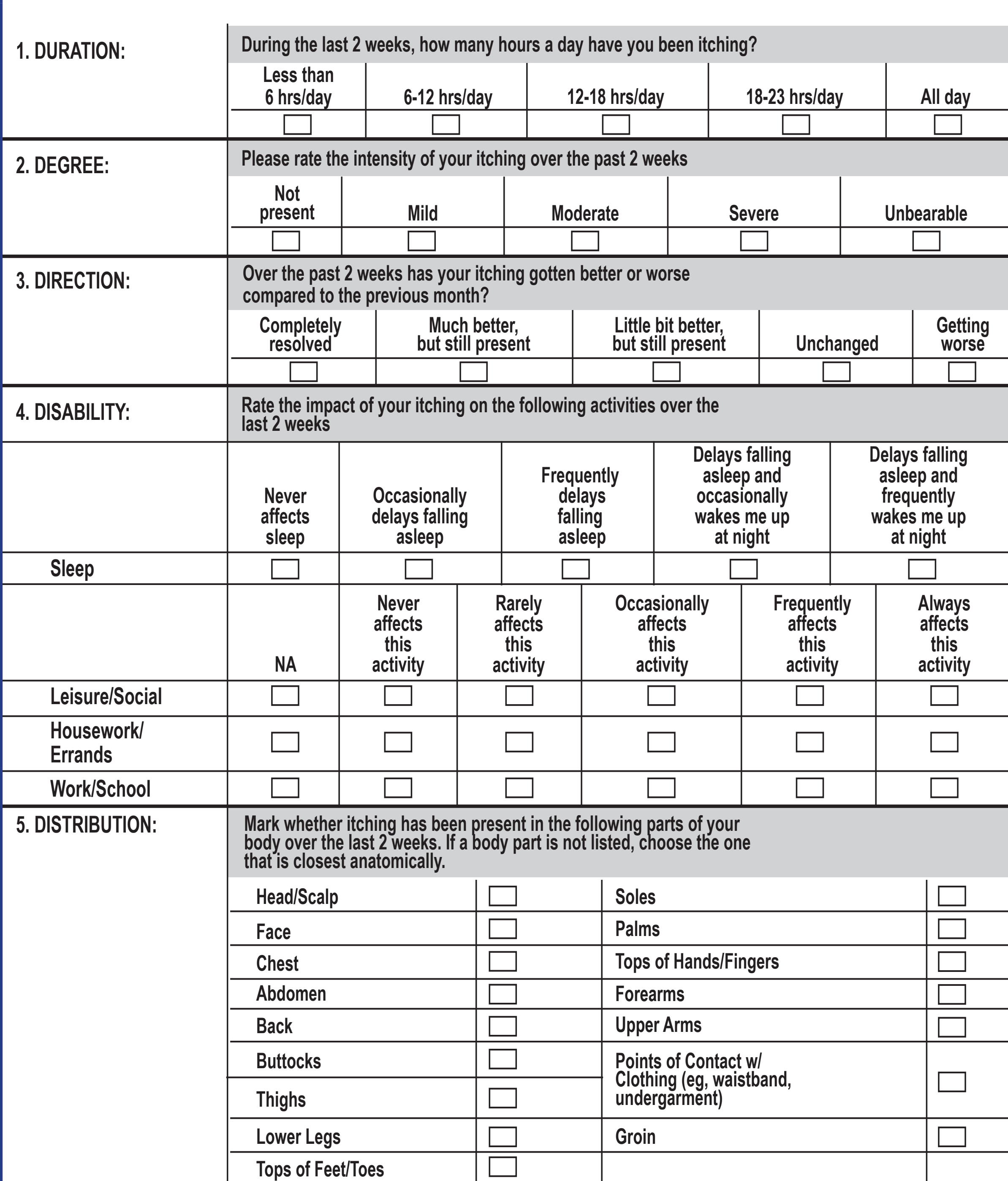

Statistical Analyses

Estimated percent, odds ratio, and P value were derived
using logistic regression with terms for treatment troup. baseline score, use of anti-tith medication during the medical conditions. A region/study combined variable was
also included in the model for the pooled analysis For the primary endpoint, missing volues were imputed using multiple imputation (MI) under missing at randor
(MAR) assumption. In the analysis of complete response. Missing values were treated as nonresponders. Skindex-10
and 5 - Dltch hesponses were analyzed without imputation
for missing values RESULTS

There were 851 randomized subjects in the pooled
KALM-1 and KALM-2 analysis (DFK: 426; placebo: 425)

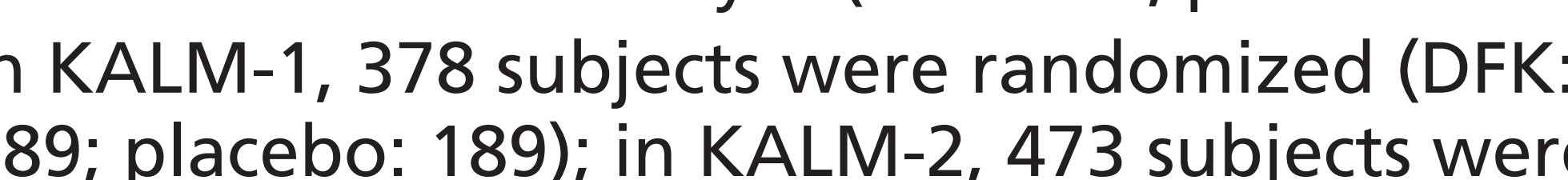

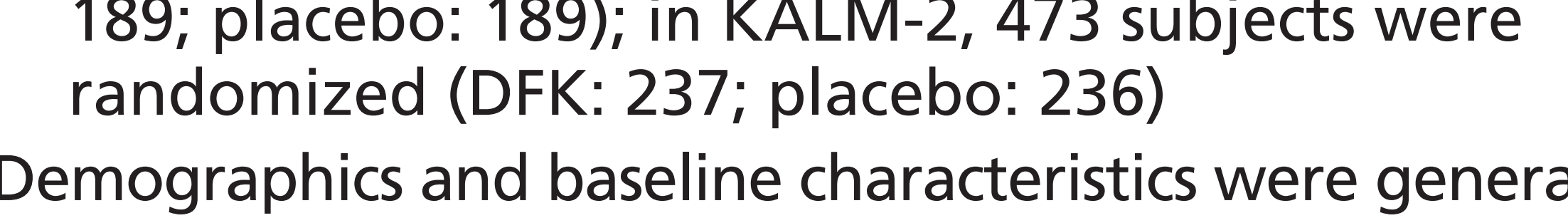
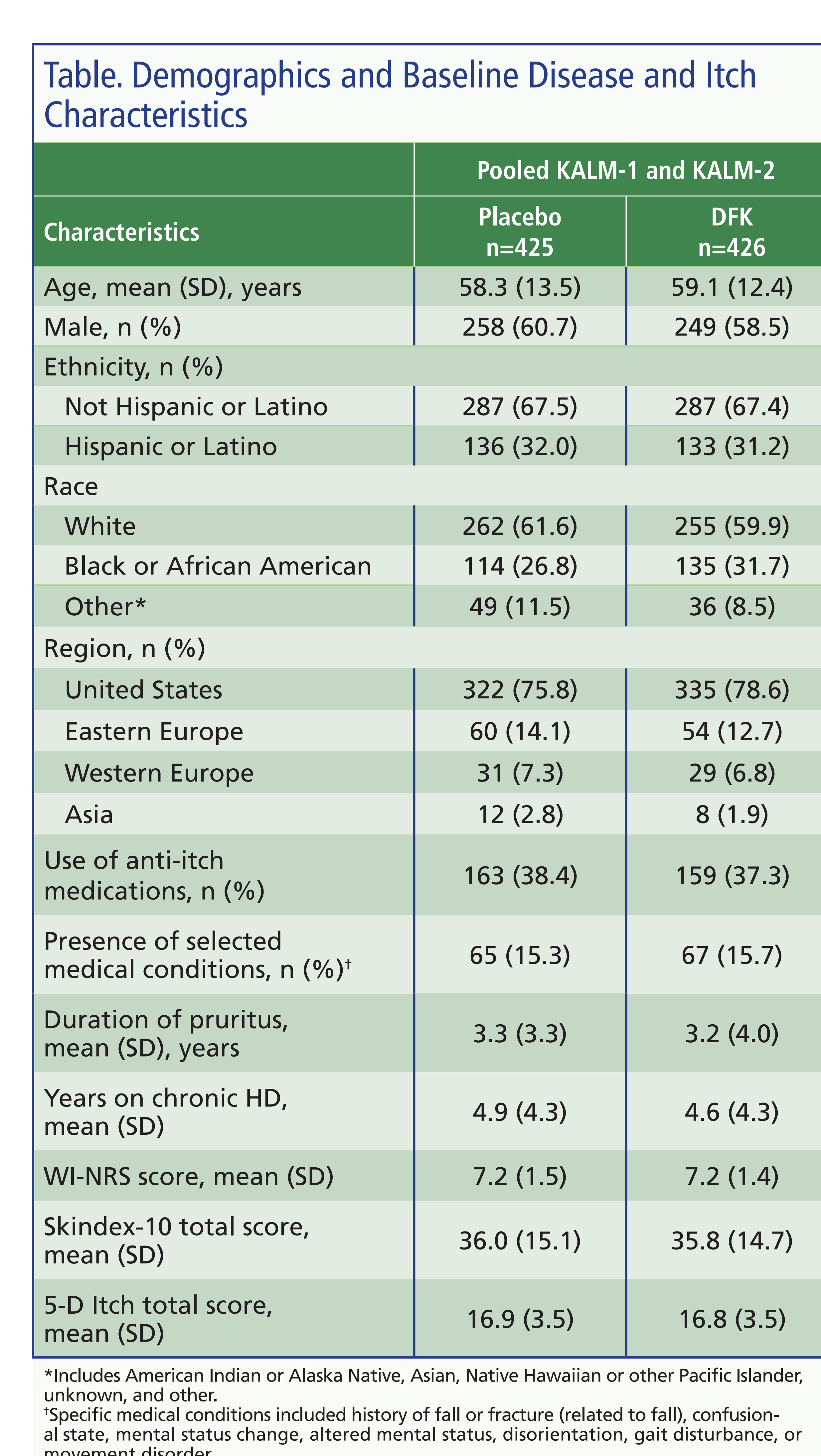

The primary endpoint, proportion of subjects achieving
23-point improvement in W-NRS score at week 12, was

KALM-1 and KALM-2, DFK vs placebo: $50.0 \%$ vs $27.6 \%$,
$P<0.001$, and $54.0 \%$ vs $42.2 \%$, $P=0.02$

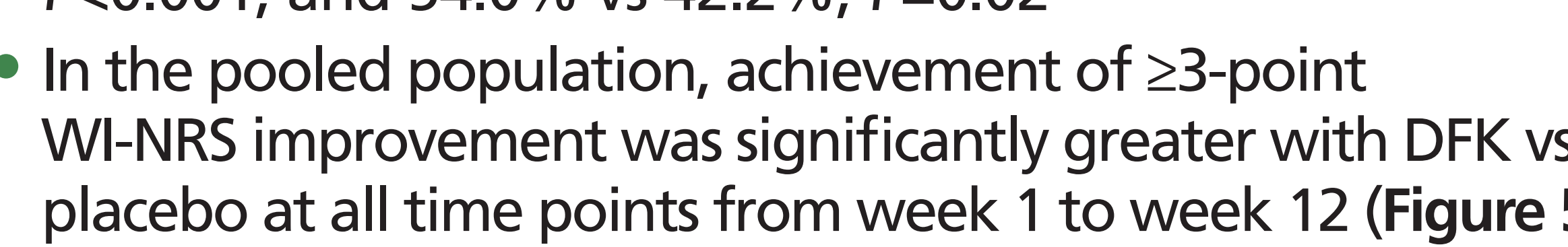

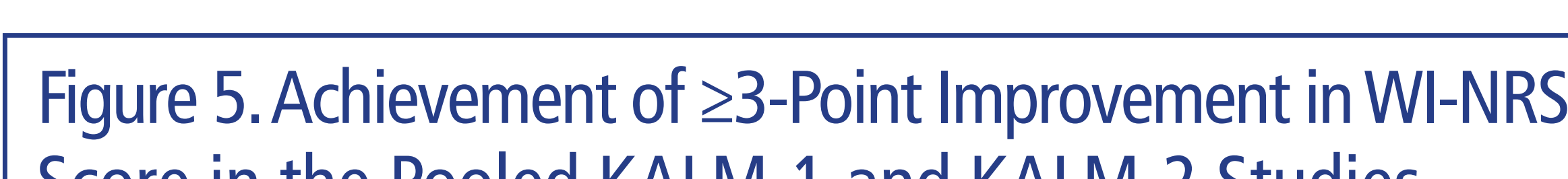

Figure 5. Achievement of 33 -Point Improvement in WW-NS
Score in the Pooled KALM-1 and KALM-2 Studies

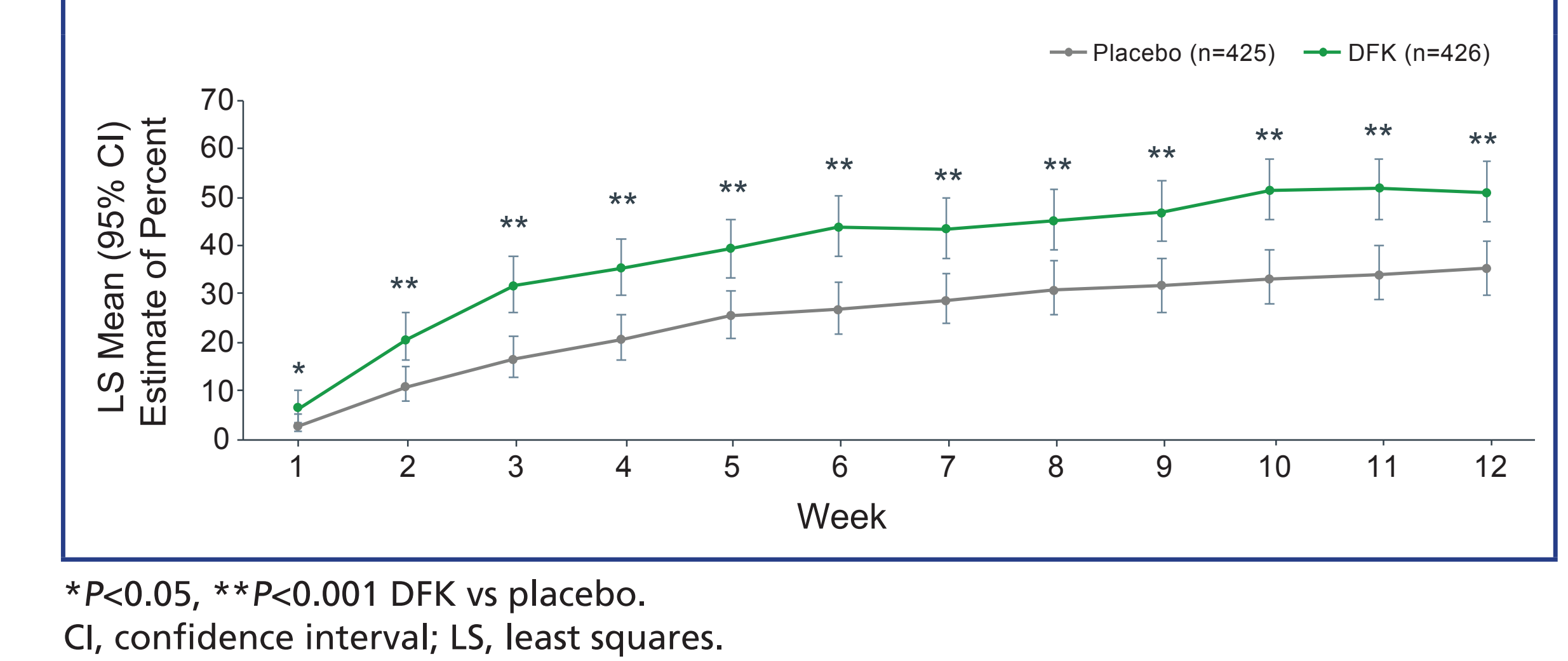

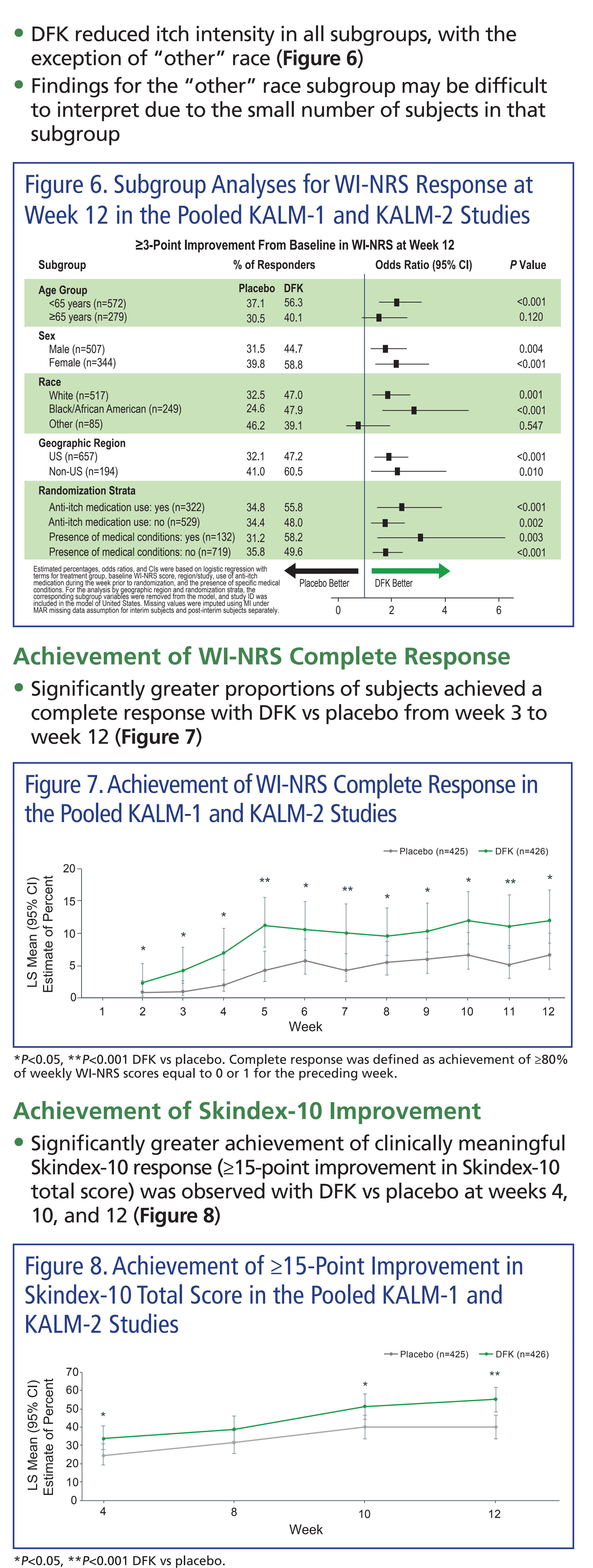

Achievement of 5-D Itch Improvement

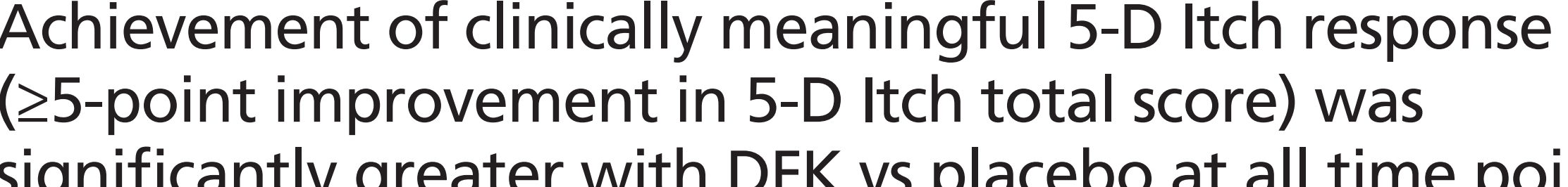
significantly greater with
up to week 12 (Figure 9 )

Figure 9. Achievement of 25 -Point Improvement in 5-D Itt
Total Score in the Pooled KALM-1 and KALM-2 Studies

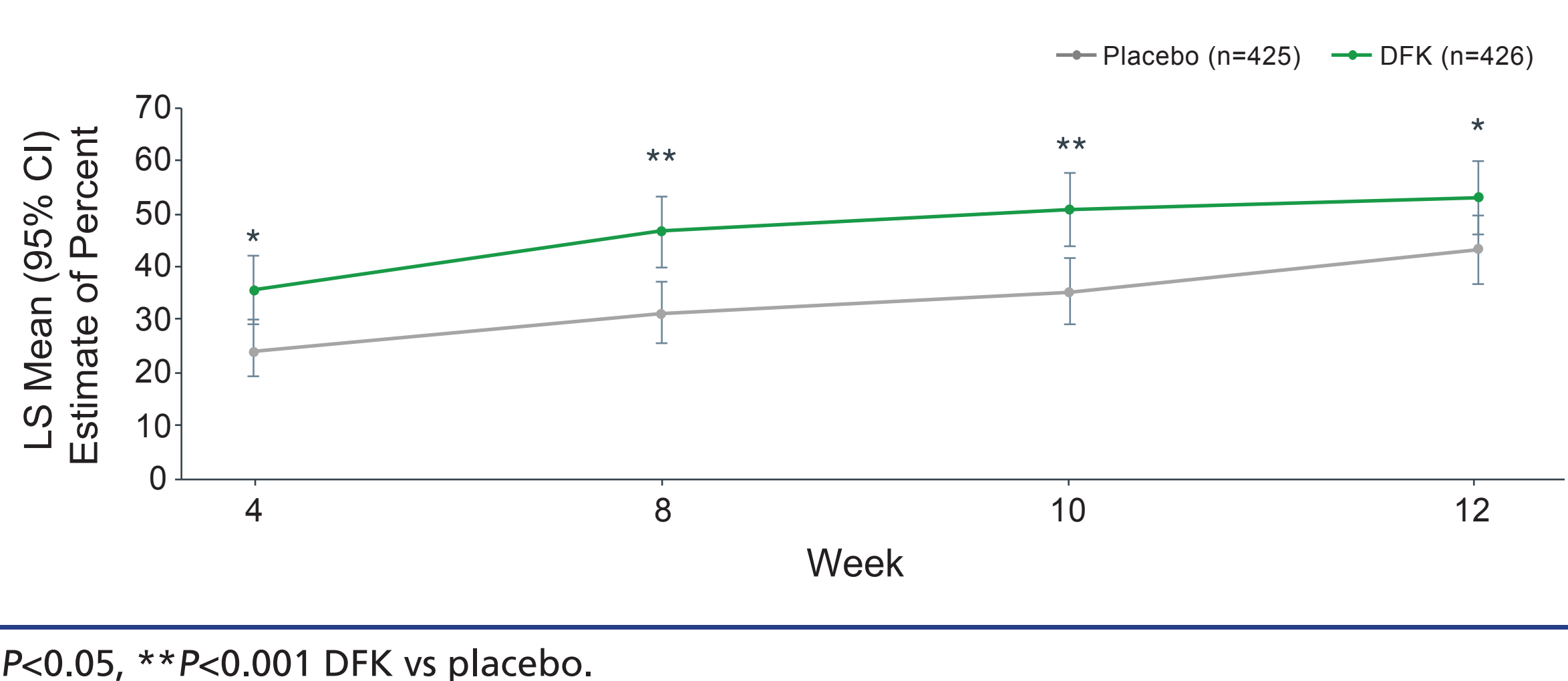

\section{CONCLUSIONS}

- In this pooled analysis of the US KALM-1 and globo
KALM-2 studies, the efficacy of IV DFK 0.5 m cog/kg Was confirmed across multiple demographic and
disease characteristic subbroups in subjects with

groups in subjects with
P undergoing HD
sceived IV DFK achieved

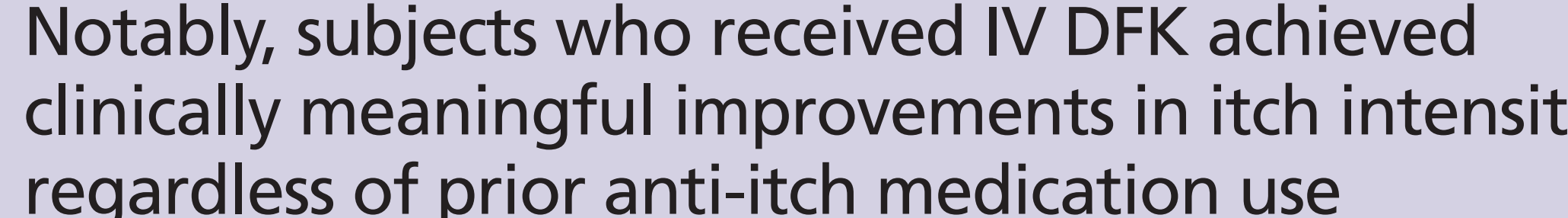
- Rates of complete response in WI-NRS were as early as week 3 in the pooled population
and maintained through week 12

population achieved clinically meaningful and
significantly greater improvements in itch-related placebo

These pooled efficacy findings suggest IV DFK may
play an important role in the care of CKD patients
undergoing HD by reducing itch and improving QoL

REFERENCES

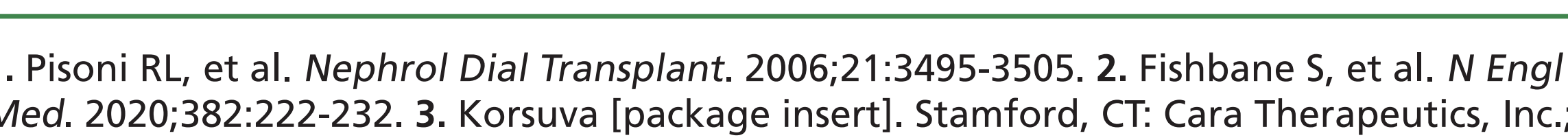

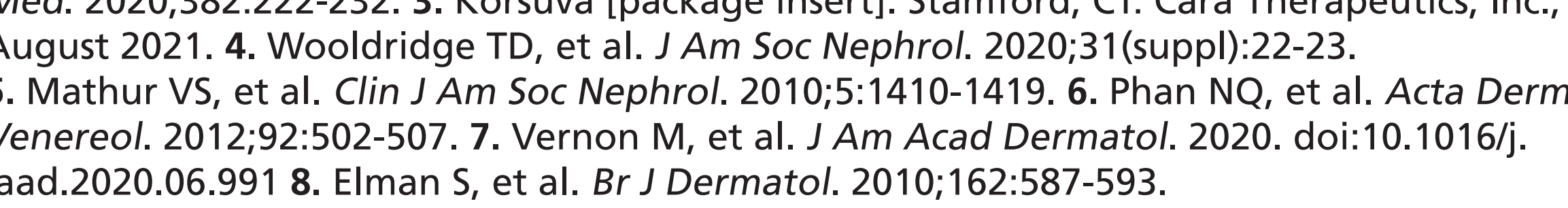
ACKNOWLEDGMENTS

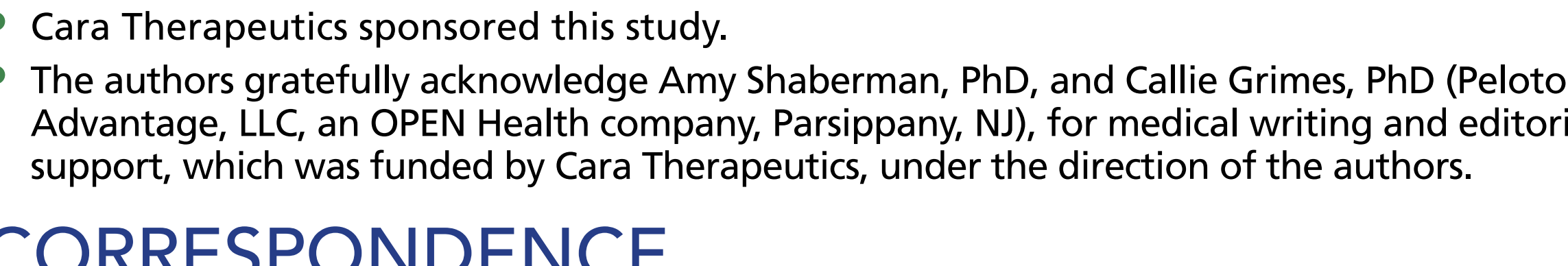
CORRESPONDENCE

DISCLOSURES

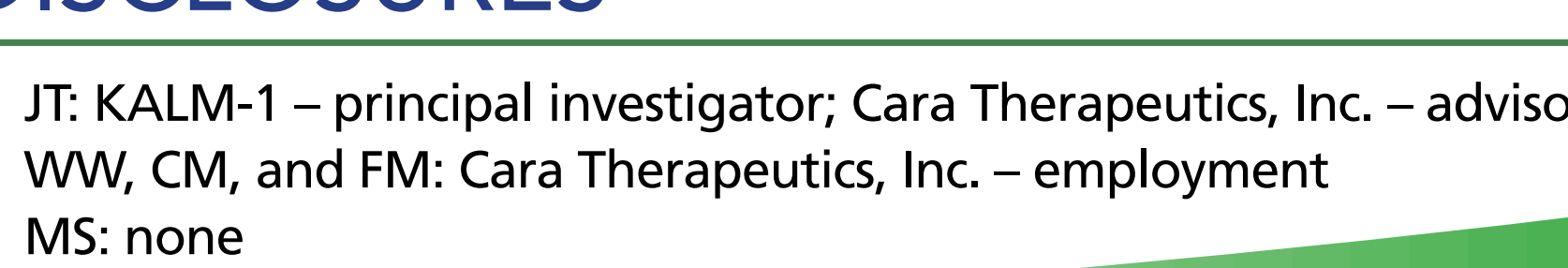

